\title{
Stickstofftantal.
}

Die durch Erhitzen von Tantalchlorid in Ammoniak entstehende schwarze Masse, welche früher wohl für metallisthes Tantal gehalten wurde, enthält Stickstoff, allein es feblt noch an weiteren Versuchen über ibre Zusammensetzung.

\section{A. Oppenheim: Mittheilung über die Wiedergewinnung von Schwefel und Manganperoxyd aus Rückständen der Sodafabrik zu Dieuze.}

Der Leblanc'sche Procefs der Sodafabrication, die Grundlage aller chemischen Industrie und die damit zusammenhängende Bereitung von Chlorkalk leiden bekanntlich an einem Uebelstande. Sie bringen bedeutende Rückstände hervor, welche Terrain einnehmen, insofern sie fest sind, oder die dem benachbarten Ackerlande and Wasser Gefahr drohen, insofern sie aus stark sauren und chlorhaltigen Flüssigkeiten bestehen. Dazu kommt, dafs die grofsen Mengen von Schwefel und Braunstein, welche in die Fabrication eintreten, sämmtlich in den Rückständen untergehn; und zwar in solchen Mengen, dafs in einer einzigen Fabrik (Dieuze), welche seit kaum $30 \mathrm{Jahren,}$ anfangs nur in beschränktem Umfang, arbeitet, der als Calciumoxysulphuret in den festen Rückständen nutzlos aufgespeicherte Schwefel auf Eine Million Thaler geschätzt wird. Endlich geben diese zuerst unlöslichen Rückstände durch allmähliche Veränderung schwefelhaltige Laugen $\mathrm{ab}$, welche die benachbarten Ländereien beschädigen können.

Es sind diese Uebelstände, welche seit einiger Zeit zu Versuchen geführt haben, entweder um den Leblanc'schen Procefs durch ander' zu ersetzen (Brisse, Gossage, Tessié du Mothay) oder um die beschwerlichen Rückstände aufzuarbeiten und zu verwerthen. Während in ersterer Hinsicht praktische Resultate noch nicht erzielt sind, haben die Versuche zweiter Art den vollständigsten Erfolg gehabt.

Es kam hier vor Allem darauf an, den Schwefelgehalt der Rückstände so zu vertheilen, dafs für ein Molecül Salzsäure, welches zu seiner Ausscheidung verwendet wird, mehr als ein Atom Schwefel in Freiheit gesetzt wird. Obwohl nämlich der Sicilische Schwefel seinen Preis behauptet, so ist der im Schwefelkiese gebundene Schwefel, welcher seit einiger Zeit in der Schwefelsäurefabrication zur Anwendung kommt (Perret's Process), aufserordentlich wohlfeil und nur za dem Kostenpreise des letzteren (welcher sich in Dieuze auf $1 \frac{1}{3} \mathrm{Thl}$. per Centner stellt) würde die Schwefelregeneration sich lohnen. 
Nehmen wir mit Dumas und E. Kopp an, dafs der unlösliche Rückstand im Anfang wesentlich aus $(\mathrm{CaS})_{2} \mathrm{CaO}$ besteht, so handelt es sich also nicht nur darum, das Schwefelcalcium löslich zu machen, sondern es auch in Polysulphuret zn verwandeln. Beides geschieht durch eine zweckmärsig geleitete Oxydation, welche einen Theil des Schwefelcalciums in Kalk verwandelt, während der freiwerdende Schwefel das übrig bleibende Schwefelcalcium in. höhere Schwefelstufen überführt, welche mit dem Kalk keine Verbindung eingehn. Dabei geht ein Theil des Schwefels allerdings in Schwefelsäure, ein anderer aber in unterschweflige Säure über und dieser letztere Umstand hat den grofsen Vortbeil, dafs bei der späteren Zersetzung der löslichen Theile des Rückstandes mittelst Salzsäure Schwefelwasserstoff nicht frei wird, sondern durch Entwicklung der schwefligen Säure aus dem Hyposulphit in Schwefel und Wasser übergeht. -

Seit 1852 haben Losh und Noble, seit 1858 Guckelberger und Schaffner diesen Theil der Aufgabe zu lösen gesucht, und Schaffner hat inn zuerst praktisch verwerthet und im Jahre 1866 8000 Centner Schwefel reproducirt ${ }^{1}$ ). Mittlerweile, um 1860 , beschäftigten sich Townsend und Walker ${ }^{1}$ ) damit, statt der verhältnilsmälsig werthvollen Salzsäure vielmehr diڤ Rückstände der Chlorkalkfabrication, die saure Chlormanganlösung (Manganbrühe) zu verwerthen. E. Kopp und P. W. Hofmann haben diese Aufgabe weiter verfolgt und sie mit mebrfachen Verbesserungen in der Fabrik zu Dieuze (Département de la Meurthe) in Frankreich vor einigen Jahren zur Lösung und grolsartigen Anwendung gebracht. -

Ich hatte den Vortheil, dies merkwürdige Verfahren durch eigene Anschauung kennen zu lernen und von dem Director der Fabrik, Herrn Dr. P. W. Hofmann für unsere Gesellschaft eine Mittheilung über die jetzt angewandte Methode zugesichert zu erhalten. Vielfach beschäftigt hat er es vorgezogen, mich durch Uebermittelung von Proben, eigenen Notizen und Berichten von Herrn Prof. Rosenstiehl an die société d'encouragement de Mulhouse vom 17. Oct. 1867 und von Herrn Bell an die British Association in den Stand zu setzen, ihn hierin, so weit es mir möglich ist, zu vertreten. -

Die folgenden Analysen der festen Rückstände nach E. Kopp (1) und der flüssigen Rückstände nach P. W. Hofmann (2) mögen der Besprechung ibres Verfahrens vorausgeschickt werden.

1) Siehe Scheurer-Kestner Sur la réproduction du soufre. Bull. Soc. ind. de Mulhouse Fevr. 1868. 
(1)

\begin{tabular}{|c|c|c|c|}
\hline $\mathrm{Na}_{2} \mathrm{~S}$ & 2,9 & $\mathrm{Mn} \mathrm{Cl}_{2}$ & 22,0 \\
\hline $\mathrm{FeS}$ & 2,7 & $\mathrm{Fe}_{2} \mathrm{Cl}_{6}$ & 5,5 \\
\hline $\mathrm{CaS}$ & 22,2 & $\mathrm{BaCl}_{2}$ & 1,1 \\
\hline $\mathrm{CaO}$ & 8,6 & $\mathrm{Cl}$ & 0,1 \\
\hline $\mathrm{CaCO} \mathrm{CO}_{3}$ & 13,6 & $\mathrm{H} \mathrm{Cl}$ & 6,8 \\
\hline $\mathrm{CaSiO} \mathrm{O}_{3}$ & 5,7 & $\mathrm{H}_{2} \mathrm{O}$ & 64,5 \\
\hline $\mathrm{Mg} \mathrm{Al} \mathrm{O}$ & 1,5 & & 100,0 \\
\hline Kohle & 1,8 & & \\
\hline $\mathrm{Cl}$ und $\mathrm{Aq}$ & 38,7 & & \\
\hline
\end{tabular}

Es ergiebt sich danach in den festen Rückständen ein Gehalt von 12 pCt. Schwefel.

Die Verarbeitung der Rückstände zu Dieuze besteht aus sieben aufeinander folgenden Processen, die wir einzeln betrachten müssen:

1) aus der Oxydation der festen Rückstände,

2) ihrer Auslaugung,

3) der Fällung von Schwefel aus den Laugen mittelst der freien Salzsäure in der Manganbrühe,

4) der Fällung des Eisens aus der letzteren als Schwefeleisen,

5) der Fällung ihres Mangangehalts als Schwefelmangan,

6) der Verbrennung des Schwefelmangans, und

7) der Verarbeitung der übrigbleibenden Asche.

1. u. 2. Die Oxydation und Auslaugung. Herr Hofmann hat gefunden, dafs die Oxydation wesentlich beschleunigt wird, wenn man den festen Rückständen Manganoxyd oder Eisenoxyd hinzufügt. Diese Substanzen gehen dabei in Sulphide über, welche ihrerseits wieder Sauerstoff nehmen und zu Oxyden werden, also den Sauerstoff der Luft an den Rückstand übertragen. -

Die alten aufgespeicherten Rückstände werden deshalb mit etwas Manganbrühe begossen und einige Tage lang der Luft ausgesetzt.

Die neuen täglich gewonnenen Rückstände dagegen werden anders behandelt, nämlich mit dem Schwefeleisen vermengt, welches bei dem Process 4. gewonnen wird. Ich mufs deshalb die Beschreibung des letzteren hier einschalten.

Man leitet die neutralisirte (nach 3. siehe unten) tägliche Ausbeute an Manganbrühe in ausgemauerte Bassins und mischt sie mit 4-5 Cubicmeter von der täglichen Ausbeute an festen Rückständen (dem fünften Theil). Man rührt um, bis fast alles Eisen niedergeschlagen ist, d. h. bis die abfiltrirte Probe mit Schwefellauge nicht mehr einen schwarzen, sondern einen grauen Niederschlag giebt. Nach einigen Stunden wird die überstehende nun fast reine Lösung von Mangan- 
chlorid abgeleitet und die eisensulphidhaltige breiförmige Masse in den Bassins mit dem Rest (20 Cubicmeter) der täglich producirten Rückstände gemengt.

Die ganze Masse wird am vierten Tage ausgeschaufelt und in regelmäfsigen Haufen eine Woche lang der Luft ausgesetzt. Dies Umlagern verhindert, dafs die Temperatur über $90^{\circ}$ steigt. Andernfalls würde zu viel schwefelsaurer Kalk entstehen, ja die ganze Masse ins Glühen gerathen können.

Man füllt sie darauf in andere Bassins mit doppeltem Boden ein. Drei derselben liegen in einer Reihe und jedes falst den festen Rückstand eines Tages und 30 Cubicmeter Wasser. Das letztere sickert durch die Masse bindurch und sammelt sich zwischen den beiden Böden an. Durch eine Röhre kann es in das zweite und von da in das dritte Bassin eingelassen werden. Aus diesem tritt es nach drei Tagen gesăttigt und etwa $50^{\circ}$ warm aus. Beim Erkalten setzen sich aus der tiefgelben Elüsrigkeit Crystalle ab, auf die Herr Hofmann in einer andern Mittheilung zurückkommen will.

Die festen Rückstände werden nun herausgenommen, noch einmal 2 Tage lang der Luft ausgesetzt und aufs Neue ausgelaugt.

Während die erste Oxydation and Auslaugung hauptsächlich Calciumpolysulphurete in Lösung bringt ( eaux jannes"), wird jetzt vorwaltend unterschwefligsaurer Kalk gelöst. Die zweite Lauge ( „eaux jaunes oxydées") wird von der ersten ( in Cysternen angesammelt. Der feste Rückstand ist nunmebr nack 14 tägiger Behandlung auf $\frac{2}{3}$ seines ursprünglichen Volums zusammengeschrumpft. Wenn man ihn zum dritten Male der Luft aussetzt, so erwärmt er sich noch schwach, weil das noch darin enthaltene Schwefeleisen in Sulphat übergeht. Aber einè neue Auslangung würde kein nutzbares Product liefern. Er kann ohne Scbaden für das Erdreich fortgeschafft werden. Seine Zusammensetzung ist nach Herrn Hofmann:

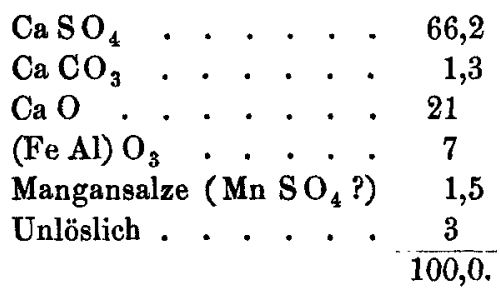

3. Die Fällung von Schwefel aus den gewonnenen Laugen geschieht mittelst der rohen durch Stehen geklärten Manganbrühe, welche auf diese Weise von ihrer freien Salzsäure und ihrem Chlor befreit wird. 
Man kann den Zufues der beiden schwefelhaltigen Laugen ( jaunes" und neaux jaunes oxydées") so reguliren, dafs bei ibrer Zersetzung kein Schwefelwasserstoff entweicht. Um aber auch bei fehlerhafter Operation von diesem Gase nicht belästigt zu werden, leitet man die Fällung in der folgenden Weise. Die beiden Laugen einerseits und die Manganbrübe andererseits werden durch Röhren zusammengeführt, aber nicht in einem offenen Bassin, sondern in einer Art von bleiernem Thurm ron 1 Meter Höhe und 0,90 Meter Durchmesser, welcher in der Mitte eines Bassins steht. Etwas über dem Punkt, wo die Röhren zusammentreffen, flielsen durch zwei Seitenöffnungen die gemischten Flüssigkeiten aus dem Thurm in das Bassin. Man beobachtet ihre Farbe. Ist dieselbe gelb, so ist saure Lösung, ist sie schwarz, so ist Lauge im Ueberschuls vorhanden und man regelt durch Hähne den Zuflufs so, dafs ein ganz geringer Ueberschufs von Lauge zugeführt and Schwefel gefüllt wird, der nur wenig Schwefeleisen beigemengt enthält.

Aus dem Dach des Thurms entweichen die etwa gebildeten Gase durch eine Röbre, die in der Mitte eines Heerdes austritt und auf welchem einige Stäcke Holz brennen. Sie werden hier bei geringem Luftzutritt unvollständig verbrannt, so dafs sich Schwefel an den Wänden des Ofens absetzt und die übrigen $G$ ase durch vorgelegte mit Wasser gefüllte Kasten streichend - welche $\mathrm{S}_{2}$ zurüekhalten aus einer Abzugsröhre entweichen. Diese Vorrichtung bat nur einen hygienischen, keinen öconomischen Vortheil. -

Der gefällte Schwefel wird mittelst Schaufeln aus den Bassins herausgeholt und getrocknet. Er beträgt $36 \mathrm{pCt}$. der in den Rückständen enthaltenen Menge. Die hellgraue Masse enthält 90 pCt. reinen Schwefel.

Die neutralisirte Manganbrühe wird durch Pumpen entfernt, um jetzt von Eisen befreit zu werden.

4. Fällung von Schwefeleisen. Dieser Procels ist beiläufig unter 1. und 2. beschrieben worden. Man leitet die somit gewonnene fast eisenfreie Lösung in mit Asphalt ausgekleidete Behälter und setzt der ganzen Menge 1 Cubikmeter gelber Lauge zu, um auch die letzten Reste des Eisens zu entfernen, damit in den folgenden Processen eisenfreies für die Glasmanufactur geeignetes Manganoxyd gewonnen werde.

5. Fällung von Schwefelmagan. Die somit nur noch Chlorcalcium und Chlormangan enthaltende Lösung fliefst in andere Behälter ab. Man lälst gelbe Lauge zutreten (nicht oxydirte Lauge, weil unterschwefligsaures Mangan löslich, also nicht fällbar ist), bis kein Niederschlag mehr statt hat. Nach 24stündigem Stehen lälst man die jetzt völlig ausgenutzte Flüssigkeit fortlaufen, zuerst in eine 
Cysterne, wo sie von Regierungswegen auf ihre Neutralität geprüft wird, und dann in den Fluls.

Das gefällte rosenrothe Mangansulphid enthält freien Schwefel. Es wird auf mit leinenen Filtern versehenen Bütten gewaschen und auf erwärmten Platten bei niederer Temperatur getrocknet. Die Gefabr der Entzündung wird umgangen, indem man die Stücke des Gemenges mit der Schaufel zerdrückt.

Dies Sulphid enthält 58,6 pCt. Schwefel, wovon $\frac{2}{3}$ in Schwefelkohlenstoff löslich, also in freiem Zustande darin enthalten ist. Der Niederschlag besteht danach aus 40 pCt. Schwefel, 55 pCt. Mn S und 5 pCt. Mn O. Es kommen somit ungefähr 3 Atome Schwefel auf 1 Atom Mangan; Beweis, dafs das Schwefelcalcium der gelben Lange hauptsächlich ans $\mathrm{Ca}_{\mathbf{3}}$ besteht. Dies schwefelhaltige Sulphid enthält 8 bis 10 pCt. von dem Schwefelgebalt der verarbeiteten Rückstände.

Es bräunt sich an der Luft, indem es, wie Herr Hofmann gefunden hat, in freien Schwefel und Manganoxyd (nicht Mangansulphat) übergebt. Hierin liegt die Gefahr der Selbstentzündung begründet, welche man in der angegebenen Weise verbütet.

Man nimmt darauf

6. die Verbrennung des schwefelbaltigen Schwefelmangans in denselben Oefer vor, die zur Verbrennung von Schwefel dienen, und leitet die schweflige Säure in die Bleikammern. Die zurückbleibende Asche besteht in Procenten aus:

$\begin{array}{ll}\mathrm{Mn} \mathrm{SO}_{4} & 44,5 \\ \mathrm{Mn} \mathrm{O}_{2} & 18,9 \\ \mathrm{Mn} \mathrm{O} & 36,6 .\end{array}$

7. Es bleibt jetzt nur noch übrig, den letzten Procers zu beschreiben, welcher in einem scharfsinnigen Verfahren besteht, um den Gehalt des Rückstandes an Manganperoxyd zu vermehren und zugleich seine Schwefelsäure in der Form von reinem Glaubersalz nutzbar zu machen.

Man mischt ihn zu diesem Zweck mit der dem Sulphat entsprechenden Menge Salpeter und erbitzt ihn in den gewöhnlichen Schwefelöfen. Dabei bildet sich Glaubersalz und salpetersaures Mangan, welches sofort in Manganperoxyd und Stickstoffsuperoxyd zerfällt:

$$
\begin{array}{ccc}
\mathrm{Mn} \mathrm{SO}_{4}+2 \mathrm{NaNO}_{3} & =\mathrm{Mn}\left(\mathrm{NO}_{3}\right)_{2}+2 \mathrm{NaSO}_{4} \\
\mathrm{Mn}\left(\mathrm{NO}_{3}\right)_{2} & = & \mathrm{MnO}_{2}+\mathrm{N}_{2} \mathrm{O}_{4}
\end{array}
$$

Die rothen Dämpfe treten in die Bleikammern ein. Das Glaubersalz wird ausgelaugt. Der Rückstand besteht ans reinen Manganoxyden, die $55 \mathrm{pCt}$. Peroxyd enthalten, also als Sauerstoffquelle dem Braunstein gleichwerthig sind, während ihre Freiheit ron Eisen ihren Werth bedeutend darüber hinaus erböht. 
Von den behandelten Rückständen ist also nun der eine um $\frac{1}{3}$ an Volum verringert, seines schädlichen Einflusses und seines Schwefelgehaltes bis zu $46 \mathrm{pCt}$. des letzteren beraubt worden. Der andere hat seinen ganzen Mangangehalt als Braunstein zurück geliefert und ist in neutrale Chlorcalciumlösung verwandelt abgeflossen.

Dafs dieses sinnreiche System trotz seiner Complication nicht nur die wissenschaftliche Lösung eines Problems, sondern ein practisches Fabricationsverfahren darbietet, geht schon daraus hervor, dafs dasselbe in Dieuze fortwährend in grofsem Maalsstabe zur Anwendung kommt. Der im Jahre 1867 daselbst wieder gewonnene Schwefel stellt einen Werth von 16000 Thalern dar. Weitere Beweise giebt durch eine genauere Besprechung der industriellen Verhältnisse der oben angeführte Bericht von Herrn Rosenstiehl. Herr P. W. Hofmann hat eine ausführliche Darstellung des Verfahrens in Aussicht gestellt.

85. A. W. Hofmann: Ueber Anilingrün

wird demnächst erscheinen.

\section{Mittheilungen.}

\section{A. Eller: Ueber Carbonaphtolsäure.}

Das Naphtol lälst sich in ganz entsprechender. Weise wie das Phenol (vergl. Ann. d. Chem. u. Pharm. 113, p. 125 u. 115, p. 177) überführen in die der Salicylsäure entsprechende Oxysäure des Naphtalins.

Die Reaction geht mit grofser Leichtigkeit vor sich und giebt eine gute Ausbeute. Das Gemenge von Naphtol mit $1 \mathrm{Mol} . \mathrm{Na}_{2}$ erhitzt sich beim Ueberleiten von trockener Kohlensäure. Wenn die Substanzen möglichst innig gemischt sind, so sublimirt nur wenig unverändertes Naphtol und findet auf dem Wasserbade unter Aufschäumen der Masse, wobei das Natrium schmilzt, die nahezu vollständige Ueberführung in das Natriumsalz der neuen Säure statt. 